\title{
The natural way to clean the oral cavity
}

As practitioners know, the build up of plaque biofilm on the surface of teeth can increase the risk of a patient developing caries or periodontitis over time. ${ }^{1}$ Therefore, the removal of bacteria and their food sources is vital to the prevention and management of oral diseases. Twice daily brushing with a fluoride toothpaste and the daily use of an interdental brush are essential to maintaining good oral hygiene.

For patients with acute dental problems or limited dexterity, the use of a mouth rinse as a supplement enables them to protect their teeth and rinse out any bacteria, when mechanical removal of these microorganisms may be particularly challenging. As a key ingredient in many current mouth rinse products, chlorhexidine is well-known for its efficacy at protecting teeth against a wide range of bacteria.

However, the long-term use of mouth rinses that contain chlorhexidine alone can result in adverse side effects such as tooth discolouration and impaired taste. ${ }^{2}$ Alcohol-based mouth rinse products can also dry out the mouth and lower saliva production, which enables oral bacteria to proliferate.

These issues, combined with current patient preferences, emphasise the need for innovative, new mouth rinses comprised of safe and highly effective ingredients. As a result, scientists have looked to mother nature in order to develop Citrox, which has the potential to revolutionise many industries - including dentistry.

\section{What is Citrox?}

Manufactured using naturally-derived, non-toxic, hypo-allergenic, and non-corrosive ingredients, Citrox takes advantage of the bioflavonoids (plant-based compounds) found in plants. Scientists have discovered that bioflavonoids have the ability to modify the body's reaction to allergens, inflammation, viruses, and carcinogens.

Not only can bioflavonoids protect the body against oxidative and free-radical damage, they can also increase the strength of the capillaries, thereby regulating their permeability to prevent ruptures and protect against infection. This helps to heal wounds and support a healthy immune system.

Citrox is created using the bioflavonoids in the dried, ground piths and pulp of bitter oranges. This is then mixed with other natural substances (organic acids) to produce a powerful antimicrobial, antioxidant, and anti-inflammatory agent. Citrox has now been developed as a key mouth rinse ingredient, as it has the ability to kill $99.9998 \%$ of bacteria, viruses, and fungi. This means that out of 10,000 potentially harmful microorganisms, only 0.1 of these could affect an individual following treatment of Citrox, making it a highly effective, natural, antimicrobial substance.

\section{How can Citrox benefit us?}

Experts at Cardiff University believe that Citrox has numerous advantages as a key ingredient in oral healthcare products, including mouth rinses. Research has revealed that Citrox is effective against dangerous pathogens such as MRSA, C. Difficile, Hepatitis A, Hepatitis B, and Streptococcus. According to a study by Williams et al., Citrox combined with other supplements is more effective than chlorhexidine alone at protecting the oral cavity from a wide range of bacteria known to cause oral diseases such as dental caries and periodontitis. This is due to the substance's ability to inactivate

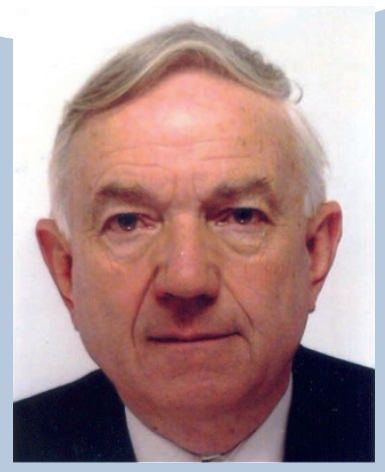

Howard Thomas, Founder and Chairman of Citrox Biosciences Ltd a variety of microorganisms by affecting multiple target sites, thereby surpassing traditional antibiotics that usually target one specific area.

Used as a vital mouth rinse ingredient, Citrox works by breaking down bacterial biofilm quickly and efficiently to ensure a fast-acting result with long-lasting, residual effects - a concentration of just $0.25 \%$ Citrox solution can kill biofilm cells in less than ten minutes. The strength of Citrox is evident even in the presence of organic matter, and it is also less prone to bacterial resistance so that it can protect the human body against bacterial re-infiltration. This makes Citrox an extremely powerful substance that can help patients maintain good oral hygiene practises that help to prevent the risk of oral health complications. ${ }^{3}$

\section{The organic option}

Patients can feel confident knowing that Citrox is completely biodegradable, safe to handle, and eco-friendly. As Citrox is alcoholfree and does not contain any synthetic fragrances or colourants, it is manufactured as a colourless and odourless solution.

This not only ensures Citrox does not change the flavour of food and drink for uninterrupted taste, but also minimises the possibility of any staining on natural teeth and dental restorations, which - as we are well aware - is typical of many mouth rinse products currently available on the market.

For additional peace of mind, Citrox is GMO (genetically modified organism) free, does not contain any petrochemicals or allergic substances, and is not tested on animals - thus providing a truly natural alternative to traditional mouth rinse ingredients.

Oral diseases remain a major concern across the world, emphasising the importance of reminding patients to maintain excellent oral hygiene at home. Using the most effective dental products is essential, particularly for patients suffering from acute dental problems.

Products that contain Citrox not only offer the benefits of a safe, natural solution, but one that has been clinically proven to protect against some of the world's most harmful microorganisms.

For more information please call 01480 862084, email info@curaprox.co.uk or visit www.curaprox.co.uk.

1. Marsh P D. Dental plaque as a biofilm and a microbial community - implications for health and disease. BMC Oral Health 2006; 6 (Suppl 1): S14.

2. Gokul G, Lakshmanan R. Effect of chlorhexidine mouthwash on taste alteration. Asian J Pharmaceut Clin Res 2016; 9 (Suppl 1): 102-104.

3. Malic S, Emanuel C, Lewis M A O, Williams D. W. Antimicrobial activity of novel mouthrinses against planktonic cells and biofilms of pathogenic microorganisms. Microbiol Discov 2013; 1: 11 